\section{$\underset{\substack{\text { hommes } \\ \text { \& migrations }}}{ }$}

\section{Hommes \& migrations}

Revue française de référence sur les dynamiques

migratoires

1307 | 2014

L'Afrique qualifiée dans la mondialisation

\title{
Retour à l'École des Annales ? Les nouveaux enjeux de l'enseignement des migrations
}

Jean-Pierre Bat

\section{(2) OpenEdition}

\section{Journals}

Édition électronique

URL : http://journals.openedition.org/hommesmigrations/2919

DOI : 10.4000/hommesmigrations.2919

ISSN : 2262-3353

Éditeur

Musée national de l'histoire de l'immigration

\section{Édition imprimée}

Date de publication : 1 juillet 2014

Pagination : 176-179

ISBN : 978-2-919040-28-5

ISSN : $1142-852 X$

\section{Référence électronique}

Jean-Pierre Bat, «Retour à l'École des Annales ? Les nouveaux enjeux de l'enseignement des migrations », Hommes \& migrations [En ligne], 1307 | 2014, mis en ligne le 16 janvier 2015, consulté le 22 septembre 2020. URL : http://journals.openedition.org/hommesmigrations/2919; DOI : https:// doi.org/10.4000/hommesmigrations.2919 


\section{RETOUR À L'ÉCOLE DES ANNALES ? LES NOUVEAUX ENJEUX DE L'ENSEIGNEMENT DES MIGRATIONS}

JEAN-PIERRE BAT, archiviste-paléographe (École nationale des Chartes),

agrégé et docteur en histoire de l'université Paris-I-Panthéon-Sorbonne, membre de l'Institut des mondes africains (IMAF)

$\mathrm{L}^{\mathrm{e}}$ es longs feux contemporains de l'histoire économique et sociale, dont l'École des Annales fut le porte-étendard, semblent avoir lentement oublié l'histoire démographique au profit de dimensions plus sociales sinon sociétales'. La modélisation démographique aurait fait montre de ses limites à "dire le réel". Paradoxalement, la démographie reste un indice pédagogique majeur. C'est sans doute là que réside une des clés du problème : appréhender la démographie, sur une histoire très contemporaine (seconde moitié du XXe siècle), non comme une science de schématisation, mais comme un indicateur humain qu'il convient d'interroger sous un nouvel angle méthodologique.

\section{La ligne Oder-Neisse, une frontière pédagogique}

Dans l'Europe de 1945, la frontière orientale de l'Allemagne vaincue, pourtant fondamentale pour I'histoire de l'Europe centrale, s'avère éclipsée au profit du rideau de fer. La géographie du fleuve Oder et de son affluent la Neisse lui a conféré son nom. Cette frontière a connu une migration forcée, entre 1944 et 1945, de millions d'individus. La mesure quantitative, qui constitue le premier enjeu, est traditionnellement présen- tée de la manière suivante : 7 millions d'émigrés en provenance de Pologne, 3 millions en provenance de Tchécoslovaquie, et entre 2 et 6 millions des autres pays d'Europe centrale - soit entre 12 et 16 millions de migrants dans l'ombre de l'effondrement de l'Allemagne nazie (dont la moitié meurt dans le courant de ces migrations). Pourquoi cet événement migratoire reste-t-il la portion congrue de l'enseignement de l'histoire de l'Europe ? Trois dimensions pédagogiques sont à prendre en considération. En premier lieu, la question du nationalisme (allemand, au premier chef) sous-tend ce phénomène migratoire. La Shoah, en second lieu (6 millions de victimes juives), bouleverse le rapport à la problématique migratoire. À ce titre, l'affaire Eichmann constitue une clé pour appréhender le glissement des approches épistémologiques du phénomène migratoire en Europe ${ }^{2}$. En troisième lieu, la dimension traumatique autant - sinon plus - que la dimension quantitative s'impose comme un nouveau paramètre fondamental, de sorte que les phénomènes migratoires revêtent une dimension de plus en plus individualisée dans leur mode d'enseignement. L'histoire démographique ne suffit donc plus à "dire" le phénomène migratoire dans l'enseignement de l'histoire. Pour autant, les migrations restent encore très largement abordées au prisme de l'immigration. Or, traditionnellement 
incluse dans la synergie des Trente Glorieuses et de la question de la main-d'œuvre, cette question se voit ainsi altérée par trois principales clés de lecture : la logique de développement des méthodes de production ; le phénomène de décoIonisation et de post-colonie ; l'enjeu Nord / Sud dans la globalisation 3 . En d'autres termes, les migrations se verraient réduites à leur expression économique à travers la question de l'immigration : née avec l'histoire démographique, la question des migrations ne peut se contenter de devenir un avatar de l'histoire économique 4 .

Il existe donc un besoin de "réinventer" I'approche du phénomène migratoire, trop souvent réduit à une géographie historique; il s'agit bel et bien de le penser comme un objet disciplinaire d'analyse politique et sociale. Cette tendance émerge à travers le cours inaugural en classe de seconde sur les migrations et le rapport de l'histoire démographique européenne dans l'histoire mondiales. Entre histoire immédiate et géopolitique, une réinvention méthodologique est en cours.

\section{Les nouvelles voies du politique dans l'Afrique de l'après-guerre froide}

Si la question migratoire dans l'Europe de la seconde moitié du XXe siècle a partiellement perdu de sa puissance comme indice du politique, il n'en est pas de même en Afrique. À l'aube des années 1990, les migrants deviennent un enjeu humanitaire et politique. Au préalable, il s'agit d'interroger la cartographie de certains pôles d'attraction : cette lecture polarisante renvoie (invisiblement) à une historicité des migrations, dégageant des perma- nences, comme l'atteste en Afrique de l'Ouest la question agricole autour de la Côte d'Ivoire ${ }^{6}$. Dans cette lecture structurée autour de la triade traditionnellement employée "migration-logique économique-attraction polaire?", forgée sur un moyen ou long terme, toute crise intervient comme un accélérateur ou un perturbateur qui, déstabilisant à court terme cette triade, invite à repolitiser l'approche du phénomène migratoire. Les grandes crises des années 1990-2000 en portent la marque : la "guerre nomade en Afrique de l'Ouest, la bande saharo-sahélienne ou encore la crise des Grands Lacs. Par-delà les conflits d'essence politique, il convient d'ajouter l'enjeu majeur de la sécurité alimentaire, notamment dans la corne de l'Afrique, qui offre une lecture politique de la sousrégion à travers ses interprétations migratoires. Dans le cas de la crise des Grands Lacs, et au regard des avancées historiographiques en histoire comme en géographie, il est tentant d'interroger l'équation suivante $:$ démographie + politique $=$ géopolitique?

Les migrations sont ici considérées comme un indice des conflits régionaux, un moyen de rendre intelligibles et plus humaines des zones de guerre, dont les tenants et les aboutissants s'avèrent toujours délicats à interpréter. Le poids de ce facteur migratoire apparaît de manière très lisible à travers les missions de l'Onu qui créent des zones humanitaires sûres (ZHS) pour protéger les populations déplacées. Les deux cas d'études les plus célèbres sont les ZHS du Darfour et de la frontière orientale du Congo. Toutefois, les ZHS agissent comme un indicateur humain et démographique, mais ne politisent pas convenablement "en soi" la lecture de ces phénomènes migratoires, comme l'a montré

3. Dans l'explication des facteurs migratoires, trois principales faiblesses peuvent être soulignées : le manque de certitudes statistiques, la distinction entre comportement personnel et comportement de groupe, et, enfin, le risque de réduire les conséquences sociomigratoires nées de la crise de 1973 à une grille de lecture pour comprendre les réactions sociétales face à la crise. 4. Bien souvent, la question des migrants et des migrations trouve, en géographie, un écho avec les subversions des frontières. La frontière américano-mexicaine en constitue l'archétype. Dans les années 2000, la cartographie de la Méditerranée (proposée au baccalauréat) a rejoint les classiques de ces études de cas. 5. Cet enseignement avait été imaginé à l'appui de la révision d'une histoire européano-centrée. Voir Jack Goody, Le Vol de l'histoire. Comment l'Europe a imposé le récit de son passé au reste du monde, Paris, Gallimard, 2010. 6. Voir notamment la cartographie de la "pression migratoire" établie par Stephen Smith dans son Atlas de l'Afrique, Paris, Autrement, 2009, pp. 56-57. 7. Dans ce processus, il convient de noter la place des centres urbains au cours des trois dernières décennies. Voir Laurent Fourchard, Gouverner les villes d'Afrique. État, gouvernement local et acteurs privés, Paris, Karthala, 2007 ; Laurent Lardeux, “Les migrants dans les villes postcoloniales d’Afrique centrale. Entre tensions et recompositions des espaces urbains", in Afrique contemporaine, vol. 1, n² 237, 2011, pp. 13-28. 8. Michel Galy, Guerres nomades et sociétés ouest-africaines, Paris, L'Harmattan, 2007. 


\section{MÉMOIRE}

la polémique née en 1994 avec la ZHS du SudOuest rwandais, puis avec les violences survenues dans les camps de Bukavu et Goma depuis 1996. Le phénomène migratoire armé qui succède au génocide rwandais procède d'une réaction en chaîne à travers l'Afrique centrale et scelle la nouvelle géopolitique régionale au lendemain de la fin de la guerre froide en Afrique ${ }^{9}$. Trois phénomènes traduisent cet "effet de souffle". En premier lieu, les migrations "militaires" (entendons armées, que ces formations soient régulières ou pas) deviennent un facteur dominant les insécurités en Afrique centrale. En second lieu apparaît le "piège ethnique ${ }^{10 "}$ si difficilement maîtrisé depuis les années 1960 et qui se surimpose, dans toute sa complexité, aux processus de démocratisation amorcés dans les années 1990. En troisième lieu vient la chute en 1997 du despote Mobutu, dinosaure de la guerre froide : la menace communiste - dont il était le rempart depuis les indépendances - disparue en 1991, ses protecteurs américains l'abandonnent et recomposent leur nouvelle géopolitique africaine depuis l'Ouganda et le Rwanda". Avec l'instauration de cette nouvelle géopolitique continentale après 1990 caractérisée par la recrudescence des activités et missions de l'Onu -, le contrôle des mouvements de populations s'est accru au nom de la sécurité humanitaire.

\section{Cartographier les migrations en Afrique : étude de cas autour de la crise malienne}

II en ressort une nouvelle appréhension "orientée" du phénomène migratoire : à trop considé- rer les migrations comme un "risque" dans la gouvernance africaine (régie en partie par les canons politiques validés à l'Onu), on oublie rapidement le caractère nomade de certaines sociétés et leur contribution à la construction de l'espace politique africain. Sous l'impulsion des bouleversements géopolitiques du continent, la nature des migrations des sociétés traditionnelles structurées par le pastoralisme évolue autant qu'elle fait évoluer les sociétés, comme le montre le cas des Mbororo entre le Tchad, le Cameroun et la Centrafrique $^{12}$. La bande saharo-sahélienne offre évidemment un exemple de choix. À titre de rappel historique peut être évoqué le franchissement de la frontière tchado-soudanaise par les hommes d'Idriss Déby en 1990, lancés à l'assaut du régime d'Hissène Habré : leur trajet épouse étroitement les circuits pastoraux traditionnels des éleveurs Zaghawa. Dans un cadre plus contemporain, la question touareg, qui agite les gouvernements malien et nigérien depuis les années 1990, offre un cas d'étude très intéressant. La tension entre la logique de la construction nationale postcoloniale (qui connait une nouvelle vitalité avec l'effort démocratique au lendemain de la reconstruction politique de 1991) et la logique du contrôle de l'espace saharo-sahélien depuis le pouvoir de Bamako a mis au second plan une donnée fondamentale : dans ce cas, la migration (nomadisme) est moins un facteur d'instabilité qu'un lieu d'équilibre et un espace d'activités souvent informelles qui mettent à mal une lecture uniquement polarisante entre une capitale et ses périphéries contrôlées.

Dans le cadre d'un croquis, l'établissement de la légende cartographique devra donc s'attacher à montrer trois dimensions. La première est

9. Voir à ce sujet la fiction littéraire de Patrick Besson, Mais le fleuve tuera l'homme blanc, Paris, Fayard, 20o9, qui permet d'aborder de manière originale l’enseignement de ce thème et de toutes les facettes de sa mémoire. 10. Jean-Pierre Chrétien, Le Défi de l'ethnisme Rwanda et Burundi (1990-1996), Paris, Karthala, 1997 ; Jean-Pierre Chrétien, Gérard Prunier, Les ethnies ont une histoire, Paris, Karthala, 2003. 11. Filip Reyntjens, La Grande Guerre africaine. Instabilité, violence et déclin de l'État en Afrique centrale (1996-2006), Paris, Le Bruit du monde, 2012. 12. Christian Seignobos, “Le phénomène zargina dans le nord du Cameroun. Coupeurs de routes et prises d'otages, la crise des sociétés pastorales mbororo", in Afrique contemporaine, vol., 3, n²39, 2011, pp. 37-59. 
l'espace saharo-sahélien et le réseau de circulation touareg face au problème de contrôle étatique : c'est dans cet espace que se développent les sanctuaires d'Aqmi et que se déploie l'activité de ses katibas. La seconde dimension correspond aux désordres politiques récents : il conviendra alors, pour éviter toute approche téléologique réductrice, de mettre en regard les rébellions touareg au Mali et au Niger. L'objet est d'en différencier le règlement politique et sécuritaire et de mettre en perspective les bouleversements de 2011-2013 qui expliquent le scénario malien. Trois éléments principaux méritent d'être ainsi mis en lumière : la rébellion de 2007-2009 au Niger qui procède d'un changement de matrice politique derrière les revendications "nationalistes touareg", l'effet de souffle de la chute du régime de Kadhafi avec l'ouverture de ses arsenaux et le retour de mercenaires touareg de Libye dès l'été 2011 (via la passe du Salvador au Niger), et le massacre d'Aguel Hoc en mars 2012 qui déclenche le putsch contre Amani Toumani Touré. La troisième dimension qui achève la dialectique des migrations et des enjeux sécuritaires est la représentation des dispositifs anti-terroristes dans la sousrégion - français (coopération militaire), américain et africain (Pan-Sahel Initiative) -, l'état de la crise sur le territoire malien avec la représentation de l'Azawad revendiqué par le Mouvement national pour la libération de l'Azawad (MNLA), d'une part, et, d'autre part, la présence et les revendications des groupes islamistes tels qu'Ansar-Dine ou le Mouvement pour l'unicité et le jihad en Afrique de l'Ouest (MUJAO). Pour être complète, cette partie doit contenir l'opération militaire internationale, dont Serval déclenchée en 2013 est le fer de lance. Au total, la recherche d'un nouvel équilibre géopolitique autour de la crise malienne a procédé au renversement des logiques "centre-périphérie" et "migration-polarisation", à travers l'accent porté sur les évolutions et les perturbations des sociétés migrantes elles-mêmes.

\section{Des phénomènes de migrations disciplinaires?}

De l'histoire à la géographie, l'enseignement des phénomènes migratoires et des migrants interroge l'approche disciplinaire individualisée autant que l'interdisciplinarité, à la lumière des outils de l'histoire immédiate et de la géopolitique, dans une dimension multiscalaire et épistémologique. Trois questionnements peuvent guider l'appréhension de l'enseignement de ce sujet. Le premier porte sur la place de l'histoire démographique comme biais pédagogique : quelle place et quelle finalité lui sont réellement accordées dans les programmes ? Le deuxième concerne l'échelle : comment passer de la dimension régionale à la dimension sociétale, et, partant, quelle est la capacité opératoire de cette démarche (car celle-ci suppose une connaissance avancée des sociétés analysées) ? Le troisième porte sur l'indice, et pourrait résumer l'emploi de l'objet "migration" dans une lecture politique : de quoi les migrations et les migrants sont-ils les indices, les révélateurs ? Or la question ne souffre aucune solution univoque, car, interrogeant du groupe à l'individu, elle ouvre sur une palette de réponses de natures aussi diverses qu'il existe de témoignages (cela permet également de multiplier les supports d'évocation des migrations à travers la démultiplication des points de vue des différents acteurs). C'est pourquoi l'analyse des indicateurs des migrations (notamment à travers les politiques de protection de populations déplacées) invite à aborder de manière politique, mais non partisane, ce sujet dans l'enseignement secondaire. C'est pourquoi aussi l'étude des migrations peut laisser une grande liberté pédagogique et méthodologique pour aborder des cas souvent négligés, quoique fondateurs du monde contemporain. 